\title{
Effects of COVID-19 Lockdown on Adherence to Individual Home- or Gym-Based Exercise Training among Women with Postmenopausal Osteoporosis
}

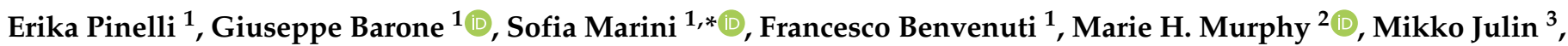 \\ Wolfgang Kemmler ${ }^{4}{ }^{\mathbb{D}}$, Simon Von Stengel ${ }^{4}$, Stefano Di Paolo ${ }^{1}$, Laura Dallolio ${ }^{5}{ }^{\circ}$, Pasqualino Maietta Latessa ${ }^{1}{ }^{1}$, \\ Raffaele Zinno ${ }^{1}$ and Laura Bragonzoni ${ }^{1}$ (D)
}

Citation: Pinelli, E.; Barone, G.; Marini, S.; Benvenuti, F.; Murphy, M.H.; Julin, M.; Kemmler, W.; Von Stengel, S.; Di Paolo, S.; Dallolio, L.; et al. Effects of COVID-19 Lockdown on Adherence to Individual Home- or Gym-Based Exercise Training among Women with Postmenopausal Osteoporosis. Int. J. Environ. Res. Public Health 2021, 18, 2441. https:// doi.org/10.3390/ijerph18052441

Academic Editor: Paul B. Tchounwou

Received: 5 February 2021

Accepted: 25 February 2021

Published: 2 March 2021

Publisher's Note: MDPI stays neutral with regard to jurisdictional claims in published maps and institutional affiliations.

Copyright: (C) 2021 by the authors Licensee MDPI, Basel, Switzerland This article is an open access article distributed under the terms and conditions of the Creative Commons Attribution (CC BY) license (https:// creativecommons.org/licenses/by/ $4.0 /)$
1 Department for Life Quality Studies, Campus of Rimini, University of Bologna, Corso d'Augusto 237, 47921 Rimini, Italy; erika.pinelli2@unibo.it (E.P.); giuseppe.barone8@unibo.it (G.B.); benvefrancis@gmail.com (F.B.); stefano.dipaolo4@unibo.it (S.D.P.); pasqualino.maietta@unibo.it (P.M.L.); raffaele.zinno2@unibo.it (R.Z.); laura.bragonzoni4@unibo.it (L.B.)

2 Centre for Exercise Medicine Physical Activity and Health, Ulster University, Newtownabbey, Co Antrim BT37 0QB, UK; mh.murphy@ulster.ac.uk

3 Department of Physiotherapy, Laurea University of Applied Sciences, 02650 Espoo, Finland; mikko.julin@laurea.fi

4 Institute of Medical Physics, Friedrich-Alexander-University Erlangen-Nürnberg, 91052 Erlangen, Germany; wolfgang.kemmler@imp.uni-erlangen.de (W.K.); simon.von.stengel@imp.uni-erlangen.de (S.V.S.)

5 Department of Biomedical and Neuromotor Science, University of Bologna, Via San Giacomo 12, 40126 Bologna, Italy; laura.dallolio@unibo.it

* Correspondence: sofia.marini2@unibo.it; Tel.: +39-051-209-4812

\begin{abstract}
Adherence is important for an exercise program's efficacy. This study aims at investigating whether the COVID-19 lockdown had different consequences on the adherence to an exercise program specifically designed for women with postmenopausal osteoporosis when administered as individual home training (IHT) or gym group training (GGT). At the start of the lockdown, which imposed the temporary closure of any gym activities, GGT participants were invited to continue to exercise at home. IHT participants continued to exercise at home as usual. Adherence was recorded via logs and measured as the percentage of exercise sessions actually performed out of the total number of scheduled sessions in three 1-month periods: one before (PRE) and two after (M1 and M2) the beginning of lockdown. Before lockdown, IHT (66.8\% \pm 26.6$)$ and GGT $(76.3 \% \pm 26.6)$ adherence were similar. During lockdown, IHT participation increased (M1: 81.5\% \pm 31.0; M2: 88.0\% \pm 28.3 ), while that of GGT showed no statistical differences (M1: 79.4\% \pm 34.2 ; M2: 80.6\% \pm 36.4 ). Exercise protocols based on supervised gym practice must consider the possibility of disruptive events, which could cause a sudden interruption of gym activity and include educational initiatives to instruct participants to exercise effectively and safely without a trainer's direct supervision.
\end{abstract}

Keywords: COVID-19; adherence; physical activity; exercise; postmenopausal osteoporosis

\section{Introduction}

Primary osteoporosis (OP) is an age-related systemic disease of the skeleton characterized by a reduced mass and deterioration of the micro-architecture of the bone, accompanied by an increased risk of fracture with consequent pain, decreased physical and social functional capacity, and quality of life (QoL) [1-9]. There is a general consensus on the efficacy of physical activity in the prevention of OP and its consequences [10,11]. A meta-analysis, which included 59 randomized controlled trials, demonstrated the efficacy of exercise programs compared to no exercise, sham programs, or pharmacological interventions in women with OP [12]. In the studies included in this meta-analysis, the exercise programs were administered either as individual home training (IHT) or in gym 
group training (GGT). In trials based on IHT, participants, after an appropriate number of educational sessions, performed exercise individually at home, without the direct supervision of a trainer, delivered to them by an exercise professional in gyms, hospitals, or community health facilities. Moreover, participants were given printed or web-based educational materials to help them in performing the requested exercises in a correct and safe way, autonomously. The time of each exercise session was chosen by the participants when they found it most convenient for them. Conversely, in the GGT, both educational activities and exercises were carried out in gym group sessions with the supervision of the trainer. The number of exercise sessions, their time, and duration were scheduled by the trainer.

Adherence to an exercise program is of fundamental importance for the programs' efficacy and is problematic in all age groups but particularly among older adults [13-15] Adherence depends on several factors, including personal-level factors and program characteristics [16]. Several personal-level factors, such as poor health, low self-confidence, low motivation, and poor enjoyment of the perceived exercise have been found to be associated with lower adherence $[15,17]$. Interestingly, reduced mental wellbeing was found to be a greater barrier to exercise adherence than reduced physical wellbeing [16]. Program characteristics also play an important role. Adherence was generally found to be higher in supervised programs than in those unsupervised, as corroborated by systematic reviews $[13,16,18-20]$. GGT could facilitate participation by enhancing social interactions, which lead to improve social, mental, and emotional health $[13,16]$. In our previous study [15], the adherence to a specifically designed exercise program was found to be the key predictor of improved back pain. Adherence, in turn, was independently associated with accessibility to gyms (shorter home-gym distance) and positive relationship with the trainer [14]. However, regular participation in GGT classes requires compliance with a fixed time schedule, which may not be compatible with family or work needs. On the other hand, IHT participants need to be well-motivated and accurately instructed to exercise autonomously.

To our knowledge, no studies compared the effects of a specific exercise program for women with postmenopausal OP when administered as IHT or GGT. Thus, our study was originally designed to consider whether IHT could be a valid alternative to GGT, since it could overcome problems related to accessibility to gyms or time schedule rigidity. With these premises, within the European project ACTLIFE, we started a randomized trial [21] to verify the efficacy of a physical activity program designed to improve the quality of life in sedentary women with postmenopausal OP, when administered IHT or GGT. During the trial, the COVID-19 pandemic resulted in a government-imposed national lockdown from 9 March-May 18 2020, restricting the movement of the population except for necessity, work, and health circumstances. The lockdown imposed the temporary closure of nonessential businesses, including gym activity, causing the interruption of the GGT but not the IHT.

This study is aimed at investigating how COVID-19 lockdown modified adherence to training practice in the two groups of ACTLIFE project's participants. We expected the adherence to exercise program of both groups to be severely disrupted during the pandemic. In addition, we hypothesized that IHT participants would be less affected by lockdown restrictions, since they were already instructed to organize their training practice autonomously at home. On the contrary, the GGT participants would have been affected to a greater extent, since they had to reorganize their weekly exercise routine and perform this individually.

\section{Materials and Methods}

When the pandemic erupted and the lockdown was imposed, a randomized controlled study was being conducted. It was aimed at investigating the efficacy of an exercise program for women with primary postmenopausal OP (T score $\leq 2.5)$, when administered as either GGT or IHT. The study was conducted within the project "Physical ACTivity: 
the tool to improve the quality of LIFE in osteoporosis people" (ACTLIFE), funded by the European Commission within the Erasmus+ Sport program (Grant Agreement N20172128/001-001). The study was conducted according to the guidelines of the Declaration of Helsinki, and approved by the Local Ethics Committee (Comitato Etico Indipendente di Area Vasta Emilia Centro) of the Emilia-Romagna Region (reference number AVEC: EM601-2019_696/2018/Sper/IOR_EM2). The trial was registered in ClinicalTrial.Gov (NCT04179903).

Details on study methods have been published previously [21]. Briefly, postmenopausal women with OP were recruited by the Centro Osteoporosi e Malattie Metaboliche dello Scheletro of Rizzoli Orthopaedic Institute of Bologna, Italy, and had no significant comorbidities affecting motor or cognitive functions. The exercise program was designed to improve quality of life in the OP population by drawing on the most recent evidence in the sector [22-24], with the aim of increasing joint mobility, muscular force, static and dynamic balance, motor coordination, and endurance. The study was a randomized trial with two parallel groups who exercised as IHT or GGT. Each group was scheduled to perform the ACTLIFE physical activity program for 12 months using simple equipment (i.e., mats, sticks, soft balls, elastic bands, weights) in two 1-h sessions per week. Moreover, all participants were requested to choose an additional third day of the week to perform brisk walking, cycling, or swimming for at least $30 \mathrm{~min}$, in order to reach the weekly amount of at least $150 \mathrm{~min}$ of exercise recommended by WHO [5]. It was a single-blinded study, since professionals who evaluated the women were not aware of to which exercise group they were assigned. Informed consent was obtained from all subjects involved in the study.

GGT was performed in two 1-h exercise sessions per week in well-equipped gyms under the direct supervision of a graduate trainer. For the IHT group, the trainer explained to the participants how to perform the physical activity at home in two 1-h unsupervised sessions per week. Participants were also given educational material with the purpose of explaining how to correctly perform the exercises. Participants were requested to strictly adhere to the instructions provided. Subsequently, the trainer contacted the IHT participants at pre-established time intervals to encourage them to exercise regularly and to obtain information on their health status. Every 6-8 weeks, a face-to-face appointment was scheduled to review and upgrade the exercise program [21], based on the progression principle.

The study protocol scheduled participants' evaluations at baseline and after 6 and 12 months. At the baseline, all participants underwent a multidimensional assessment, which included age, body mass index, functional capacity (Short Physical Performance Battery $[25,26]$ ), fear of falling (Short Fall Efficacy Scale-International $[27,28]$ ), and OPrelated quality of life (Assessment of Health-Related Quality of Life in Osteoporosis [29,30]).

Immediately after the beginning of the lockdown, IHT participants were invited to continue their weekly practice as instructed. Those in the GGT group were asked to perform the exercises learned during gym classes at home, with the support of educational material that was sent them via e-mail. For both groups, the trainers kept in touch with all participants, providing instructions and advice by telephone or video calls. Specifically, the participants were instructed to exercise in two 1-h sessions per week.

Participants were requested to record the execution of each exercise session on specific weekly logs, which had been given them by research team. Logs were returned after the end of lockdown. Adherence was measured as the percentage of exercise sessions actually performed out of the total number of planned exercise sessions in three 1-month periods: one before (PRE) and two after (M1 and M2) the date of the beginning of lockdown.

\subsection{Participants' Characteristics}

When the lockdown was imposed by the Italian national government, 48 postmenopausal women with OP had been participating in the study, but 5 did not fill or return their weekly logs (two in IHT and three in GGT group). Therefore, we included 23 women of the IHT group and 20 of the GGT group in these findings. The time interval from the beginning of the ACTLIFE exercise program and that of the lockdown was $6.8 \pm 1.5$ (range 5.0-9.6) months 
for the IHT group and $6.6 \pm 1.6$ (range 5.0-8.4) months for the GGT group (Student $t$-test $p>$ $0.05)$. No statistically significant difference was observed between the two groups at baseline assessment (Table 1). Only $49 \%$ of participants had completed the first 6 months of the study at the beginning of the lockdown.

Table 1. Characteristics of the IHT and GGT groups at the baseline assessment and adherence before the lockdown.

\begin{tabular}{|c|c|c|c|c|}
\hline & $\begin{array}{l}\text { IHT Group } \\
\quad(\mathbf{N}=23)\end{array}$ & $\begin{array}{l}\text { GGT Group } \\
\qquad(\mathrm{N}=20)\end{array}$ & Test & $p^{*}$ \\
\hline & Mean $( \pm \mathrm{SD})$ & Mean $( \pm \mathrm{SD})$ & & \\
\hline Age (years) & $65.6( \pm 5.6)$ & $65.0( \pm 7.4)$ & Student $t$ & NS \\
\hline \multirow[t]{2}{*}{ Body Mass Index } & $23.5( \pm 3.1)$ & $23.6( \pm 4.1)$ & Student $t$ & NS \\
\hline & Median (IQR) & Median (IQR) & & \\
\hline $\begin{array}{l}\text { Short Physical Performance } \\
\text { Battery }\end{array}$ & $11.0(10.0-12.0)$ & $11.0(9.0-12.0)$ & $\begin{array}{c}\text { Mann- } \\
\text { Whitney }\end{array}$ & NS \\
\hline $\begin{array}{l}\text { Short Fall Efficacy } \\
\text { Scale-International }\end{array}$ & $8.0(7.0-9.0)$ & $8.0(7.0-9.2)$ & $\begin{array}{l}\text { Mann- } \\
\text { Whitney }\end{array}$ & NS \\
\hline $\begin{array}{c}\text { Assessment of } \\
\text { Health-Related Quality of } \\
\text { Life in Osteoporosis }\end{array}$ & $1.6(1.3-1.8)$ & $1.6(1.3-2.1)$ & $\begin{array}{l}\text { Mann- } \\
\text { Whitney }\end{array}$ & NS \\
\hline
\end{tabular}

Note: GGT: group gym training group; IHT: individual home training group; SD: standard deviation; IQR: interquartile range; ${ }^{*} \mathrm{NS}: p>0.05$.

\subsection{Statistical Analysis}

The Shapiro-Wilk test was used to check the normal distribution of the data. Normal distributed continuous variables were presented as mean and standard deviation (SD), while non-normal distributed variables were presented as median and interquartile range (IQR). Categorical variables were presented as a percentage over the total. The repeated measure ANOVA test was performed to assess the between-group differences of continuous variables, along with the two times assessment, while the two-tailed Student's $t$-test was used to compare each group with one another. The Mann-Whitney U test was used to compare two groups in case of non-normally distributed variables. Differences between the groups were considered statistically significant if $p<0.05 . p$-values were adjusted using the Bonferroni post hoc correction for multiple comparisons. A post hoc power analysis was conducted in $G^{*}$ Power 3.1.9.4 (Franz Paul, Kiel, Germany) to ensure the statistical effectiveness of the results obtained. A minimum power of 0.82 was ensured, accounting for a type I error of 0.05. All statistical analyses were performed in JASP (JASP Team, 2020, Version 0.14.1 (Computer software)).

\section{Results}

As shown in Figure 1 and Table 2, adherence before lockdown (PRE) did not differ between the two groups. On the other hand, as shown in Table 3, IHT adherence showed a statistically significant progressive increase from PRE $(66.8 \pm 37.6 \%)$ to M1 $(81.5 \pm 31.0 \%)$ and to $\mathrm{M} 2(88.0 \pm 28.3 \%)$. Conversely, in the GGT group, adherence did not statistically change from PRE $(76.3 \pm 26.6 \%)$ to M1 $(79.4 \pm 34.2 \%)$ and M2 (80.6 $\pm 32.8 \%)$. 


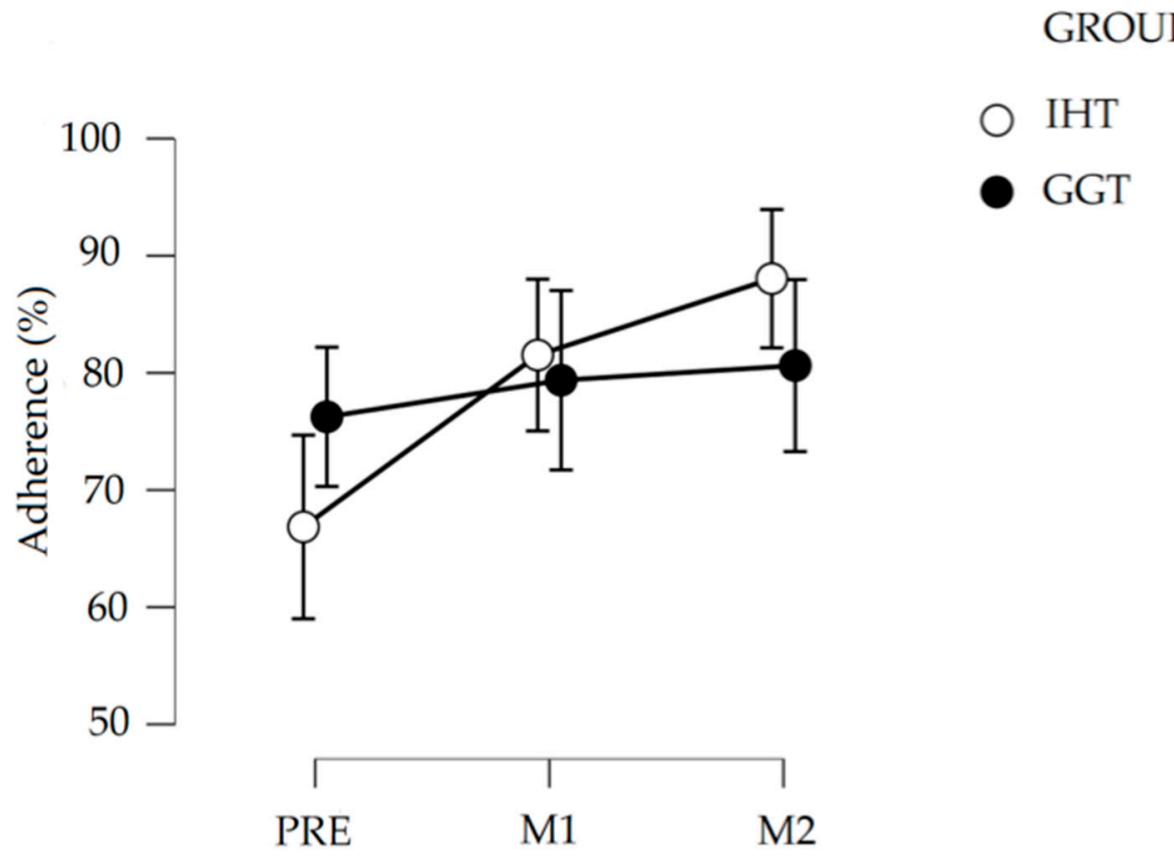

Time

Figure 1. Average percentage of adherence in a three-month period: before (PRE) and one (M1) and two (M2) months after the beginning of lockdown (8 March 2020). The error bars represent the SD.

Table 2. Repeated measure ANOVA.

\begin{tabular}{cccc}
\hline & F & $p$ & $\eta^{2} p$ \\
\hline TIME & 13.781 & $<0.001$ & 0.252 \\
TIME $\times$ GROUP & 5.936 & 0.015 & 0.126 \\
\hline
\end{tabular}

Note: Type III Sum of Squares.

Table 3. Post hoc comparison-GROUP * TIME.

\begin{tabular}{cccc}
\hline & & Mean Difference & $p_{\text {bonf }}$ \\
\hline \multirow{2}{*}{ IHT, PRE } & GGT, PRE & -9.402 & NS \\
& IHT, M1 & -14.674 & $<0.001$ \\
& GGT, M1 & -12.527 & $\mathrm{NS}$ \\
& IHT, M2 & -21.196 & $<0.001$ \\
GGT, PRE & GGT, M2 & -13.777 & $\mathrm{NS}$ \\
& IHT, M1 & -5.272 & $\mathrm{NS}$ \\
& GGT, M1 & -3.125 & $\mathrm{NS}$ \\
IHT, M1 & IHT, M2 & -11.793 & $\mathrm{NS}$ \\
& GGT, M2 & -4.375 & $\mathrm{NS}$ \\
& GGT, M1 & 2.147 & $\mathrm{NS}$ \\
GGT, M1 & IHT, M2 & -6.522 & $\mathrm{NS}$ \\
& GGT, M2 & 0.897 & $\mathrm{NS}$ \\
IHT, M2 & IHT, M2 & -8.668 & $\mathrm{~N}$ \\
& GGT, M2 & -1.250 & $\mathrm{NS}$ \\
& GGT, M2 & 7.418 & $\mathrm{NS}$
\end{tabular}

Note: $p$-value adjusted through Bonferroni correction; GGT: group gym training group; IHT: individual home training group; before (PRE) and one (M1) and two (M2) months after the beginning of lockdown (8 March 2020).

\section{Discussion}

Participation in and adherence to a program is important to the internal validity of a study, but about $50 \%$ of people who embark on an exercise program will drop out within six months [31]. Structured or group programs to increase physical activity in older 
adults have demonstrated high short-term participation rates and good long-term retention rates [31].

The COVID-19 pandemic and associated restrictions may have disrupted the study participants' routines and motivation, as staying at home can lead to reduced physical activity and sedentary behavior. In addition, the reduction or lack of social bonds that are usually essential to encourage the elderly to exercise may have reduced.

This study was aimed at evaluating the effects of the COVID-19 lockdown on training practice in postmenopausal women with OP who were administered a specific exercise program as either IHT or GGT. We investigated the adherence to the exercise protocol as the primary outcome measure since, as stated above, it is of fundamental importance to prove its efficacy and validate the expected results [13-15]. In general, attending an exercise training program regularly is a challenge, as several factors can serve as barriers to exercise adherence $[15,16,32]$. In women with postmenopausal OP, adherence to an exercise protocol may also be prevented by fear of falls and fracture [33]. In this scenario of expected low participation, we expected the adherence to drop even more during lockdown, given the great uncertainty caused by the COVID-19 pandemic [34-36]. On the contrary, we observed that adherence of both groups at least maintained the levels recorded before the lockdown. With present methods, we cannot empirically prove the reason for this observation. However, we speculate that this result might be due to the educational activity performed within the study to promote an active lifestyle among participants and/or to the increased free time compared to the normal daily routines without lockdown. The ongoing relationship between participants and trainer during lockdown via telephone and social media may have facilitated adherence, as suggested by previous studies [37-40].

The present study also hypothesized that IHT adherence would have been less affected by lockdown restrictions than that of the GGT, since participants were already instructed to organize their training practice autonomously at home. Indeed, we found a marked increase in weekly training practice in the initial lockdown months in IHT but not in the GGT group. This result endorses the notion of the importance of specific educational strategies to promote a more active lifestyle oriented to the prevention of chronic diseases and their consequences [41].

\section{Conclusions}

Results lead us to conclude that exercise protocols, even if based on supervised gym practice, must consider the possibility that a disruptive event (or, more simply, a change in a person's daily routine) could cause a sudden interruption of gym participation. Therefore, it is very important to include in the exercise protocols educational approaches to instruct participants to exercise effectively and safely without the direct supervision of a trainer. This needs to be supported by the ongoing relationship and supervision of trainers, which may be facilitated by telephone or other appropriate technological tools [37-40].

Author Contributions: E.P. conceived the study; L.B., G.B., and S.M. contributed to the study project and drafted the manuscript; R.Z. and F.B. contributed to data analysis and interpretation of results; S.D.P. performed statistical analysis; L.D., P.M.L., M.H.M., M.J., S.V.S., and W.K. supervised the study. All authors provided feedback and approved the final manuscript.

Funding: This research was funded within the ACTLIFE project by Erasmus+ Sport (grant agreement N2017-2128/001-001).

Institutional Review Board Statement: The study was conducted according to the guidelines of the Declaration of Helsinki, and approved by the Local Ethics Committee (Comitato Etico Indipendente di Area Vasta Emilia Centro) of the Emilia-Romagna Region (reference number AVEC: EM6012019_696/2018/Sper/IOR_EM2). The trial was registered in ClinicalTrial.Gov (NCT04179903).

Informed Consent Statement: Informed consent was obtained from all subjects involved in the study.

Data Availability Statement: The data presented in this study are available on request from the corresponding author. The data are not publicly available due to ethical and privacy reasons. 
Acknowledgments: The authors acknowledge Ludovica Baldone for language revision.

Conflicts of Interest: The authors declare no conflict of interest. The funders had no role in the design of the study; in the collection, analyses, or interpretation of data; in the writing of the manuscript, or in the decision to publish the results.

\section{References}

1. Buchner, D.M.; Wagner, E.H. Preventing Frail Health. Clin. Geriatr. Med. 1992, 8, 1-18. [CrossRef]

2. Guralnik, J.M. Understanding the Relationship Between Disease and Disability. J. Am. Geriatr. Soc. 1994, 42, 1128-1129. [CrossRef]

3. Singh, M.A.F. Exercise Comes of Age: Rationale and Recommendations for a Geriatric Exercise Prescription. J. Gerontol. Ser. A Biol. Sci. Med. Sci. 2002, 57, M262-M282. [CrossRef]

4. Stuart, M.; Chard, S.; Benvenuti, F.; Steinwachs, S. Community exercise: A vital component to healthy aging. Healthcarepapers 2008, 10, 23-28. [CrossRef]

5. World health organization. Guidelines on Physical Activity and Sedentary Behaviour; World Health Organization: Geneva, Switzerland, 2020.

6. Peck, W.A. Consensus development conference: Diagnosis, prophylaxis, and treatment of osteoporosis. Am. J. Med. 1993, 94, 646-650. [CrossRef]

7. Bessette, L.; Ste-Marie, L.-G.; Jean, S.; Davison, K.S.; Beaulieu, M.; Baranci, M.; Bessant, J.; Brown, J.P. The care gap in diagnosis and treatment of women with a fragility fracture. Osteoporos. Int. 2007, 19, 79-86. [CrossRef] [PubMed]

8. Group WS. Prevention and management of osteoporosis. In WHO Technical Report Series; World Health Organization: Geneva, Switzerland, 1994.

9. Choi, M.; Prieto-Merino, D.; Dale, C.; Nüesch, E.; Amuzu, A.; Bowling, A.; Ebrahim, S.; Casas, J.P. Effect of changes in moderate or vigorous physical activity on changes in health-related quality of life of elderly British women over seven years. Qual. Life Res. 2013, 22, 2011-2020. [CrossRef]

10. Beck, B.R.; Daly, R.M.; Singh, M.A.F.; Taaffe, D.R. Exercise and Sports Science Australia (ESSA) position statement on exercise prescription for the prevention and management of osteoporosis. J. Sci. Med. Sport 2017, 20, 438-445. [CrossRef] [PubMed]

11. Daly, R.M.; Via, J.D.; Duckham, R.L.; Fraser, S.F.; Helge, E.W. Exercise for the prevention of osteoporosis in postmenopausal women: An evidence-based guide to the optimal prescription. Braz. J. Phys. Ther. 2019, 23, 170-180. [CrossRef]

12. Pinheiro, M.B.; Oliveira, J.; Bauman, A.; Fairhall, N.; Kwok, W.; Sherrington, C. Evidence on physical activity and osteoporosis prevention for people aged 65+ years: A systematic review to inform the WHO guidelines on physical activity and sedentary behaviour. Int. J. Behav. Nutr. Phys. Act. 2020, 17, 1-53. [CrossRef] [PubMed]

13. Rivera-Torres, S.; Fahey, T.D.; Rivera, M.A. Adherence to Exercise Programs in Older Adults: Informative Report. Gerontol. Geriatr. Med. 2019, 5, 2333721418823604. [CrossRef]

14. Morgan, F.; Battersby, A.; Weightman, A.L.; Searchfield, L.; Turley, R.; Morgan, H.; Jagroo, J.; Ellis, S. Adherence to exercise referral schemes by participants-what do providers and commissioners need to know? A systematic review of barriers and facilitators. Bmc Public Health 2016, 16, 227. [CrossRef]

15. Hicks, G.E.; Benvenuti, F.; Fiaschi, V.; Lombardi, B.; Segenni, L.; Stuart, M.; Pretzer-Aboff, I.; Gianfranco, G.; Macchi, C. Adherence to a Community-based Exercise Program Is a Strong Predictor of Improved Back Pain Status in Older Adults: An Observational Study. Clin. J. Pain 2012, 28, 195-203. [CrossRef]

16. Picorelli, A.M.A.; Pereira, L.S.M.; Pereira, D.S.; Felício, D.; Sherrington, C. Adherence to exercise programs for older people is influenced by program characteristics and personal factors: A systematic review. J. Physiother. 2014, 60, 151-156. [CrossRef] [PubMed]

17. Lee, L.-L.; Arthur, A.; Avis, M. Using self-efficacy theory to develop interventions that help older people overcome psychological barriers to physical activity: A discussion paper. Int. J. Nurs. Stud. 2008, 45, 1690-1699. [CrossRef] [PubMed]

18. Courneya, K.S.; Karvinen, K.H.; McNeely, M.L.; Campbell, K.L.; Brar, S.; Woolcott, C.G.; McTiernan, A.; Ballard-Barbash, R.; Friedenreich, C.M. Predictors of Adherence to Supervised and Unsupervised Exercise in the Alberta Physical Activity and Breast Cancer Prevention Trial. J. Phys. Act. Health 2012, 9, 857-866. [CrossRef]

19. Lacroix, A.; Kressig, R.W.; Muehlbauer, T.; Gschwind, Y.J.; Pfenninger, B.; Bruegger, O.; Granacher, U. Effects of a Supervised versus an Unsupervised Combined Balance and Strength Training Program on Balance and Muscle Power in Healthy Older Adults: A Randomized Controlled Trial. Gerontology 2016, 62, 275-288. [CrossRef]

20. Pavey, T.; Taylor, A.; Hillsdon, M.; Fox, K.; Campbell, J.; Foster, C.; Moxham, T.; Mutrie, N.; Searle, J.; Taylor, R. Levels and predictors of exercise referral scheme uptake and adherence: A systematic review. J. Epidemiol. Community Health 2012, 66, 737-744. [CrossRef]

21. Bragonzoni, L.; Barone, G.; Benvenuti, F.; Canal, V.; Ripamonti, C.; Marini, S.; Dallolio, L. A Randomized Clinical Trial to Evaluate the Efficacy and Safety of the ACTLIFE Exercise Program for Women with Post-menopausal Osteoporosis: Study Protocol. Int. J. Environ. Res. Public Health 2020, 17, 809. [CrossRef]

22. Giangregorio, L.M.; Papaioannou, A.; MacIntyre, N.J.; Ashe, M.C.; Heinonen, A.; Shipp, K.; Wark, J.; McGill, S.; Keller, H.; Jain, R.; et al. Too Fit to Fracture: Exercise recommendations for individuals with osteoporosis or osteoporotic vertebral fracture. Osteoporos. Int. 2014, 25, 821-835. [CrossRef] [PubMed] 
23. Marini, S.; Leoni, E.; Raggi, A.; Sanna, T.; Malavolta, N.; Angela, B.; Latessa, P.M.; Dallolio, L. Proposal of an Adapted Physical Activity Exercise Protocol for Women with Osteoporosis-Related Vertebral Fractures: A Pilot Study to Evaluate Feasibility, Safety, and Effectiveness. Int. J. Environ. Res. Public Health 2019, 16, 2562. [CrossRef]

24. American College of Sport Medicine. Exercise Testing and Prescritions, 5th ed.; Walter Kluver: Philadelphia, PA, USA, 2018.

25. Guralnik, J.M.; Ferrucci, L.; Pieper, C.F.; Leveille, S.G.; Markides, K.S.; Ostir, G.V.; Studenski, S.; Berkman, L.F.; Wallace, R.B. Lower Extremity Function and Subsequent Disability: Consistency Across Studies, Predictive Models, and Value of Gait Speed Alone Compared with the Short Physical Performance Battery. J. Gerontol. Ser. A 2000, 55, M221-M231. [CrossRef] [PubMed]

26. Guralnik, J.M.; Simonsick, E.M.; Ferrucci, L.; Glynn, R.J.; Berkman, L.F.; Blazer, D.G.; Scherr, P.A.; Wallace, R.B. A short physical performance battery assessing lower extremity function: Association with self-repoted disability and prediction of mortality and nursing home admuission. J. Gerontol. Med. Sci. 1994, 49, 85-94. [CrossRef] [PubMed]

27. Yardley, L.; Beyer, N.; Hauer, K.; Kempen, G.; Piot-Ziegler, C.; Todd, C. Development and initial validation of the Falls Efficacy Scale-International (FES-I). Age Ageing 2005, 34, 614-619. [CrossRef]

28. Ruggiero, C.; Mariani, T.; Gugliotta, R.; Gasperini, B.; Patacchini, F.; Nguyen, H.; Zampi, E.; Serra, R.; Dell'Aquila, G.; Cirinei, E.; et al. Validation of the Italian version of the falls efficacy scale international (FES-I) and the short FES-I in community-dwelling older persons. Arch. Gerontol. Geriatr. 2009, 49, 211-219. [CrossRef]

29. Badia, X.; Diez-Perez, A.; Lahoz, R.; Lizan, L.; Nogués, X.; Iborra, J. The ECOS-16 questionnaire for the evaluation of health related quality of life in post-menopausal women with osteoporosis. Heal. Qual. Life Outcomes 2004, 2, 41. [CrossRef] [PubMed]

30. Salaffi, F.; Malavolta, N.; A Cimmino, M.; Di Matteo, L.; Scendoni, P.; Carotti, M.; Stancati, A.; Mulé, R.; Frigato, M.; Gutierrez, M.; et al. Validity and reliability of the Italian version of the ECOS-16 questionnaire in postmenopausal women with prevalent vertebral fractures due to osteoporosis. Clin. Exp. Rheumatol. 2007, 25, 390-403.

31. Cox, K.; Burke, V.; Gorely, T.; Beilin, L.; Puddey, I. Controlled Comparison of Retention and Adherence in Home- vs CenterInitiated Exercise Interventions in Women Ages 40-65 Years: The S.W.E.A.T. Study (Sedentary Women Exercise Adherence Trial). Prev. Med. 2003, 36, 17-29. [CrossRef]

32. Macías-Hernández, S.I.; Loya-García, F.; Zepeda-Mora, R.; Nava-Bringas, T.I.; Morones-Alba, J.D. Disease-related factors associated with exercise adherence in postmenopausal women with osteoporosis. J. Frailtysarcopenia Falls 2020, 5, 72-78. [CrossRef]

33. Rodrigues, I.B.; Armstrong, J.J.; Adachi, J.D.; MacDermid, J.C. Facilitators and barriers to exercise adherence in patients with osteopenia and osteoporosis: A systematic review. Osteoporos. Int. 2017, 28, 735-745. [CrossRef] [PubMed]

34. Mattioli, A.V.; Sciomer, S.; Cocchi, C.; Maffei, S.; Gallina, S. Quarantine during COVID-19 outbreak: Changes in diet and physical activity increase the risk of cardiovascular disease. Nutr. Metab. Cardiovasc. Dis. 2020, 30, 1409-1417. [CrossRef]

35. Schwartz, H.; Har-Nir, I.; Wenhoda, T.; Halperin, I. Staying physically active during the COVID-19 quarantine: Exploring the feasibility of live, online, group training sessions among older adults. Transl. Behav. Med. 2021. [CrossRef]

36. Suzuki, Y.; Maeda, N.; Hirado, D.; Shirakawa, T.; Urabe, Y. Physical Activity Changes and Its Risk Factors among CommunityDwelling Japanese Older Adults during the COVID-19 Epidemic: Associations with Subjective Well-Being and Health-Related Quality of Life. Int. J. Environ. Res. Public Health 2020, 17, 6591. [CrossRef] [PubMed]

37. Vandelanotte, C.; Spathonis, K.M.; Eakin, E.G.; Owen, N. Website-Delivered Physical Activity Interventions. Am. J. Prev. Med. 2007, 33, 54-64. [CrossRef]

38. Norman, G.J.; Zabinski, M.F.; Adams, M.A.; Rosenberg, D.E.; Yaroch, A.L.; Atienza, A.A. A Review of eHealth Interventions for Physical Activity and Dietary Behavior Change. Am. J. Prev. Med. 2007, 33, 336-345. [CrossRef]

39. Krebs, P.; Prochaska, J.O.; Rossi, J.S. A meta-analysis of computer-tailored interventions for health behavior change. Prev. Med. 2010, 51, 214-221. [CrossRef]

40. Schoeppe, S.; Alley, S.; Van Lippevelde, W.; Bray, N.A.; Williams, S.L.; Duncan, M.J.; Vandelanotte, C. Efficacy of interventions that use apps to improve diet, physical activity and sedentary behaviour: A systematic review. Int. J. Behav. Nutr. Phys. Act. 2016, 13, 1-26. [CrossRef]

41. Pennucci, F.; De Rosis, S.; Murante, A.M.; Nuti, S. Behavioural and social sciences to enhance the efficacy of health promotion interventions: Redesigning the role of professionals and people. Behav. Public Policy 2019, 1-21. [CrossRef] 